\title{
Adaptive Volterra Filter for Parallel MRI Reconstruction
}

\author{
Haifeng Wang ${ }^{1 * \dagger}$, Yihang Zhou ${ }^{2 *+} \mathbb{D}$, Shi Su ${ }^{1+}$, Zhanqi Hu ${ }^{3^{*}}$, Jianxiang Liao ${ }^{3^{*}}$ and Yuchou Chang ${ }^{4}$
}

\begin{abstract}
Parallel magnetic resonance imaging (MRI) technique is able to accelerate MRI speed for reducing costs and enhancing patient's comfortability. Parallel MRI can be categorized into two types: image-based and k-space-based methods. For k-space-based parallel MRI, missing k-space data is reconstructed by interpolating existing acquired k-space data with appropriate coefficients, which is generally considered as a linear process. However, noise cannot be suppressed or removed during the linear reconstruction process and therefore reconstructed image often suffers serious noise, especially when the acceleration factor is high. Non-linear filters are known to remove non-linear noise better. Based on the Volterra series that discovers and removes the second-order non-linear noise, we proposed a non-linear reconstruction strategy called adaptive Volterra generalized autocalibrating partial parallel acquisition (AV-GRAPPA) to reconstruct the unacquired $k$-space signals. For the proposed AV-GRAPPA, optimal selection of the second-order Volterra series terms is adjusted and determined for optimizing reconstruction quality. Experimental results show that the proposed method is able to better remove the reconstruction noise and suppress aliasing artifacts.
\end{abstract}

Keywords: Parallel MRI, GRAPPA, Second-order non-linear noise, Volterra series, Non-linear filter

\section{Introduction}

Magnetic resonance imaging (MRI) [1-3] is a non-invasive imaging technique. Different from computed tomography and other imaging technologies, MRI has the advantages of non-ionizing radiation, multiple parameter imaging, high contrast, etc. It has become an important diagnostic tool in clinical imaging. However, MRI has its own shortcomings, such as long scan time, which limit its application in many clinical situations. Therefore, investigators have been exploring rapid magnetic resonance imaging methods since the invention of MRI technology. This has led to the development of methods for spiral acquisition, radial acquisition, parallel imaging, and so forth. Those methods have greatly improved the imaging speed to some extent. In

\footnotetext{
*Correspondence: hf.wang1@siat.ac.cn; yihang.zhou@outlook.com; huzhanqi1983@aliyun.com; liaojianxiang@vip.sina.com Haifeng Wang, Yihang Zhou, and Shi Su are co-first and equal authors. +Haifeng Wang, Yihang Zhou and Shi Su contributed equally to this work. 'Paul C. Lauterbur Research Center for Biomedical Imaging, Shenzhen Institutes of Advanced Technology, Chinese Academy of Sciences, Shenzhen 518055, Guangdong, China

${ }^{2}$ Medical Physics and Research Department, Hong Kong Sanatorium and Hospital, 2 Village Road, Happy Valley, Hong Kong SAR, China

${ }^{3}$ Department of Neurology, Shenzhen Children's Hospital, Shenzhen 518038, Guangdong, China

Full list of author information is available at the end of the article
}

MRI, the speed of imaging is very important. Early MRI scan often took several hours, and then the speed of imaging has increased dramatically due to improvements in field strength, gradient, and pulse sequence. However, rapid field gradients and high-density continuous radio frequency (RF) pulses will result in higher specific absorption rate (SAR) that is unsustainable by the physiological limits of organ tissues in the human body. Therefore, the imaging speed cannot increase further.

With the application of complex computer image reconstruction algorithms with a phased array coils, imaging speed of MRI can be greatly improved. This technique is often referred to as parallel imaging technology. Parallel imaging includes simultaneous acquisition of spatial harmonics (SMASH) [4], sensitivity encoding (SENSE) [5] parallel acquisition technique, and generalized autocalibrating partially parallel acquisitions (GRAPPA) [6]. Parallel MRI (pMRI) reconstruction is an image reconstruction technique for rapid acquisition. It utilizes the spatial sensitivity difference of phased array coils for spatial encoding and simultaneous acquisition with phased array coils to achieve fast imaging speed. Currently, the acceleration in clinical applications can be about 2-6 times faster or even higher imaging speed. To use parallel imaging technology, 
new requirements are needed for MRI systems, such as multiple receiver channels, multiple array coils, and coil sensitivity calibration.

SMASH is a parallel acquisition and reconstruction method that uses coil sensitivity to fit spatial harmonic functions and fills undersampled data. The characteristics of the algorithm include the summation of the data of all channels and fitting procedure. There is a large error in the fitting calculation of the traditional algorithm, resulting in a serious artifact and a low signal-to-noise ratio (SNR) of the SMASH image. Subsequently, GRAPPA enhances SMASH technique. GRAPPA uses the sampling data of all channels for fitting and recovers in the undersampled data of each channel. Then fully reconstructed image of each channel after the fitting is combined. Final image is calculated with sum-of-square on all channel images. The GRAPPA algorithm reduces the calculation error of fitting procedure and improves image quality.

When the number of acquired lines and the convolution kernel size change, GRAPPA reconstruction results also vary (corresponding to the estimation error that varies). For example, auto-calibration signal (ACS) lines are usually sampled in the central region of $k$-space (low-frequency region) rather than outer $k$-space (high-frequency region), based on which the fitting weights are derived. Therefore, interpolation would be inaccurate when they are used for reconstructing missing points at outer $k$-space region. Although variable density sampling strategy [7] has been proposed to solve this problem to some extent, estimation error still exists since it is impossible to fully sample $k$-space for reconstruction (otherwise, it is meaningless for pMRI). For both errors, Nana et al. proposed the cross-validation model for estimating coefficients [8]. Since $k$-space signals cannot be fully acquired for GRAPPA reconstruction, model error is difficult to be reduced. During the matrix inversion process [9], measured noise will be propagated and amplified in the fitting and interpolation procedures. In this paper, we focus on how to reduce the second kind of error: noise-related error. Furthermore, some works [10-13] on combining parallel imaging with compressed sensing and low-rank algorithms have been proposed. Reconstruction quality and imaging speed have been greatly enhanced.

Although some methods, including regularization-based method $[14,15]$ and iterative reweighted least-squares method [16], reduce the noise level in matrix inversion process, they fail to consider the noise generation routine in GRAPPA reconstruction. We analyze it and discover that non-linear noise is generated in the propagation process. Finite impulse response (FIR) model is currently used in GRAPPA reconstruction [17, 18]. It models GRAPPA as a linear filter. The linear filter cannot remove non-linear noise. This article presents a new methodadaptive Volterra GRAPPA (AV-GRAPPA)-to address the poor SNR problem in GRAPPA. Based on the theory of adaptive Volterra series, adaptive Volterra filter can be used for suppressing non-linear noise. The first part of the paper introduces the existing problems. The second section presents the background. The third part gives the proposed method. Experimental results are provided in the fourth part. Conclusion is given in the fifth part.

\section{Theory}

GRAPPA reconstruction can be generalized as a fitting and interpolation processes in the following equation [6]:

$$
\begin{aligned}
S_{j}\left(k_{y}+r \cdot \Delta k_{y}, k_{x}\right) & =\sum_{l=1}^{L} \sum_{b=-N_{b}}^{N_{a}} \sum_{h=-H_{l}}^{H_{r}} w_{j, r}(l, b, h) \\
& \times S_{l}\left(k_{y}+b \cdot R \cdot \Delta k_{y}, k_{x}+h \cdot \Delta k_{x}\right)
\end{aligned}
$$

where $S$ is $k$-space signal, $w$ denotes weight coefficient set, $R$ represents reduction factor, $j$ is the target coil, $l$ counts all coils, $b$ and $h$ are transverse neighbor acquired points. Indices $k_{x}$ and $k_{y}$ count through frequency encoding and phase encoding directions, respectively.

The weight coefficients are calculated by least-squares:

$$
\hat{\mathbf{x}}=\min _{\mathbf{x}}\|\mathbf{b}-\mathbf{A x}\|^{2}
$$

Generally, the errors in the inverse process can be reduced in regularization SENSE and GRAPPA reconstruction $[16,17]$ :

$$
\hat{\mathbf{x}}=\min _{\mathbf{x}}\|\mathbf{b}-\mathbf{A x}\|^{2}+\lambda\|\mathbf{x}\|^{2}
$$

where $\lambda$ is regularization parameter. If noise can be suppressed with keeping a low level of aliasing artifacts, regularization strategy is actually effective for reconstruction. Otherwise, aliasing artifacts will deteriorate image quality seriously even noise can be reduced to some extent.

Because the linear filter has a well-established theoretical basis, simple mathematical analysis, and easy design and implementation, it has been widely used. However, it is found that in the presence of non-linearity such as satellite links, high-speed communication channels, and echo cancelation, the linear filter's performance is not ideal due to the inherent disadvantages of the linear adaptive filter. In order to overcome the shortcomings of linear filters and improve system performance, non-linear filter theory research has gradually become popular. In recent years, a variety of non-linear adaptive filtering methods have been proposed, such as morphological filter, homomorphic filter, order statistics filter, Volterra filter, and other polynomial filters. Volterra filter [19-21] studies both of the linear structure and non-linear structure of the system. It is suitable for constructing non-linear models of various systems and has broad application prospects. In actual 
application, the system features are unknown or timevarying, and parameters of the filter cannot be known in advance. Non-linear active noise control (ANC) plays an important role in the field of non-linear signal processing. The non-linear characteristics contained in the Volterra filter are suitable for creating a non-linear system model and it can effectively realize non-linear active noise control. Therefore, the non-linear active noise control based on Volterra filter may become feasible for adaptive signal processing in parallel MRI reconstruction.

The flowchart of the proposed method is presented in Fig. 1. Given the noisy input signal samples $\mathbf{A}$ and noisy desired output signal samples $\mathbf{b}$, we are trying to estimate the true model parameters $x$, which is driven by na oiseless input signal $\mathbf{A}^{\prime}$ and produced noiseless output signal b'. Volterra series has been widely used in signal processing, which was developed for modeling non-linear behavior [20]. Conventional GRAPPA can be considered as a linear and time-invariant system via convolution formula for the output $v(t)$ in terms of the input $z(t)$ :

$$
v(t)=\int s(\tau) z(t-\tau) d \tau
$$

where $z(t)$ and $v(t)$ represent the input and output, respectively, and $s(t)$ is the impulse response of the system. A non-linear system is characterized by the Volterra series as

$$
v(t)=s_{0}+\int_{n=1}^{\infty} \ldots \int s_{n}\left(\tau_{1}, \tau_{2}, \ldots, \tau_{n}\right) z\left(t-\tau_{1}\right) \ldots z\left(t-\tau_{n}\right) d \tau_{1} \ldots d \tau_{n}
$$

where $s_{n}\left(\tau_{1}, \tau_{2}, \ldots, \tau_{n}\right)$ are called Volterra series coefficients. In mathematics, Volterra series represent an expansion of dynamics, non-linear, and time-invariant function. Volterra series can describe the input and output transfer characteristics of a non-linear system, which is also referred to as Volterra filter. The major drawback of Volterra filter is that a large number of weights are needed to be computed which deteriorates computation cost. Furthermore, if $n=1$ as shown in Eq. (5), it is a linear filter.

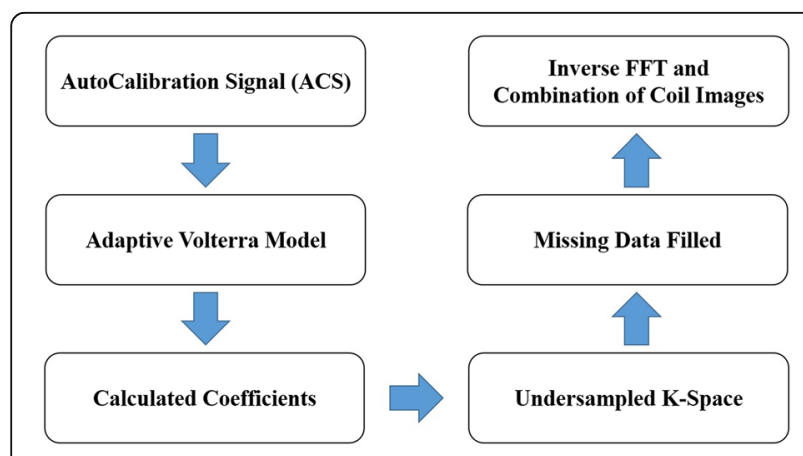

Fig. 1 Flowchart of the proposed method
GRAPPA is essential a fitting and interpolation problem, which needs data consistency to obtain optimal fitting weights. If weights are more accurate, reconstructed missing $k$-space signals are more accurate. Otherwise, serious noise and artifacts will be appearing in the reconstructed image. The proposed adaptive Volterra filter-based GRAPPA reconstruction is presented as the following equation

$$
\begin{aligned}
S_{j}\left(k_{y}+\right. & \left.r \cdot \Delta k_{y}, k_{x}\right)=w_{j, r}^{(0)}+\sum_{l=1}^{L} \sum_{b=-N_{b}}^{N_{a}} \sum_{h=-H_{l}}^{H_{r}} w_{j, r}^{(1)}(l, b, h) \\
& \times S_{l}\left(k_{y}+b \cdot R \cdot \Delta k_{y}, k_{x}+h \cdot \Delta k_{x}\right) \\
& +\sum_{l=1}^{L} \sum_{b=-N_{b}}^{N_{a}} \sum_{h=-H_{l}}^{H_{r}} \sum_{l^{\prime}=1}^{L} \sum_{b^{\prime}=-N_{b}}^{N_{a}} \sum_{h^{\prime}=-H_{l}}^{H_{r}} \\
& {\left[w_{j, r}^{(2)}\left(l, b, h, l^{\prime}, b^{\prime}, h^{\prime}\right) \times S_{l}\left(k_{y}+b \cdot R \cdot \Delta k_{y}, k_{x}+h \cdot \Delta k_{x}\right)\right.} \\
& \left.\cdot S_{l^{\prime}}\left(k_{y}+b^{\prime} \cdot R \cdot \Delta k_{y}, k_{x}+h^{\prime} \cdot \Delta k_{x}\right)\right]
\end{aligned}
$$

where $w$ denotes weight set, which has constant, firstorder, and second-order parts, respectively. The firstorder part of the equation is equivalent to the conventional GRAPPA that linearly combine the data acquired from both phase $\left(k_{y}\right)$ and frequency $\left(k_{x}\right)$ directions from all coils. The constant and second-order parts of Eq. (6) suppress the second-order non-linear noise. Noting that this relation is non-linear in terms of input of acquired $k$-space data, but linear in terms of the weights $w$, it is possible to calculate the weights in matrix form similar to Eq. (2), which can simplify the process of solving weights. After the missing $k$-space, data are reconstructed, existing ACS lines and acquired lines are used to replace the corresponding reconstructed $k$-space data for the final sum of squares reconstruction.

A large computation cost is needed to solve the weights presented in Eq. (6), which is unbearable if all second-order terms are calculated. Truncated Volterra series representation via random and pseudorandom inputs has been proposed for identifying the non-linear system [21]. In order to enhance the reconstruction speed, only a subset of the second-order terms that are randomly selected takes part in computing the weights. So, AV-GRAPPA can be reformulated as follows:

$$
\begin{aligned}
& S_{j}\left(k_{y}+r \cdot \Delta k_{y}, k_{x}\right)=w_{j, r}^{(0)}+\sum_{l=1}^{L} \sum_{b=-N_{b}}^{N_{a}} \sum_{h=-H_{l}}^{H_{r}} w_{j, r}^{(1)}(l, b, h) \\
& \quad \times S_{l}\left(k_{y}+b \cdot R \cdot \Delta k_{y}, k_{x}+h \cdot \Delta k_{x}\right)+\sum_{t=1}^{U} w_{j, r}^{(2)}(t) \times S_{p t} \times S_{q t}
\end{aligned}
$$

where $S_{p t}$ and $S_{q t}$ are randomly selected from 


$$
\begin{aligned}
& S_{l}\left(k_{y}+b \cdot R \cdot \Delta k_{y}, k_{x}+h \cdot \Delta k_{x}\right) ; l=1, \cdots, L ; b \\
& \quad=-N_{b}, \cdots, N_{a} ; h=-H_{l}, \cdots, H_{r}
\end{aligned}
$$

The reconstruction quality also depends on the choice of the total number of the second-order terms. When the number of terms $U$ is too small, reconstructed image has low SNR, and if the number of terms were too large, aliasing artifacts would appear with high SNR. Similar to the choice of convolution kernel size for optimal reconstruction result, the number of the second-order terms can also be chosen appropriately to achieve good reconstruction quality.

\section{Methods}

The proposed method is evaluated on one phantom dataset and two in vivo brain datasets. The Gaussian noise is added in this phantom dataset for testing performance. Informed consent was obtained from the volunteer in accordance with the institutional review board policy. Sensitivity information of each coil was obtained by pre-scanning. Based on empirical observation of multiple reconstructions, we choose $4 \times 7$ convolution kernel size. Furthermore, we choose 64, 48, and 38 ACS lines for the phantom, four-channel brain, and eightchannel brain data sets, respectively. For the proposed AV-GRAPPA, the number of the second-order terms is set as 672,784 , and 896 for the phantom, four-channel brain, and eight-channel brain data sets, respectively.

The proposed AV-GRAPPA is also compared to reconstructed images by Tikhonov regularization [14] and iterative reweighted least-squares (IRLS) [16] for performance evaluation. For visual evaluation, apart from directly comparing reconstructed images, difference maps and local patches are also used for comparison. Furthermore, with sum of squares (SoS) as the gold standard, reconstructions are also compared quantitatively in terms of the normalized mean squared error (NMSE) [22], which is defined as the normalized difference square between the reconstructed image $\left(I_{\text {estimated }}\right)$ and the SoS as the gold standard $\left(I_{\text {standard }}\right)$ :

$$
\mathrm{NMSE}=\frac{\left.\sum_{\mathbf{r}}|| I_{\text {estimated }}(\mathbf{r})|-| I_{\text {standard }}(\mathbf{r})\right|^{2}}{\sum_{\mathbf{r}}\left|I_{\text {standard }}(\mathbf{r})\right|^{2}}
$$

When the value of NMSE is larger, the quality of the reconstructed image is deteriorated more seriously, which suggests both increased image artifacts and noise.

For two comparison methods that reduce the noise level, Tikhonov regularization method for GRAPPA has an automatic mechanism to tune the parameter $\lambda$. Following the increasing of $\lambda$, the noise of reconstructed image will gradually disappear and aliasing artifacts will emerge. If both noise and aliasing artifacts exist in the content of the image simultaneously, the regularization does not have the power to reconstruct high-quality image, since regardless of decreasing or increasing $\lambda$, reconstructed image is always deteriorated by noise or aliasing artifacts. We manually tune the parameter $\lambda$ for Tikhonov regularization method to observe the gradual changing of reconstruction results. For IRLS, two implementations: slow robust GRAPPA and fast robust GRAPPA were proposed. Authors stated that both robust GRAPPAs have the similar performance, so we choose slow robust GRAPPA for the comparison without having to choose "outlier ratio" for different data sets, which should be done in the fast robust GRAPPA implementation.

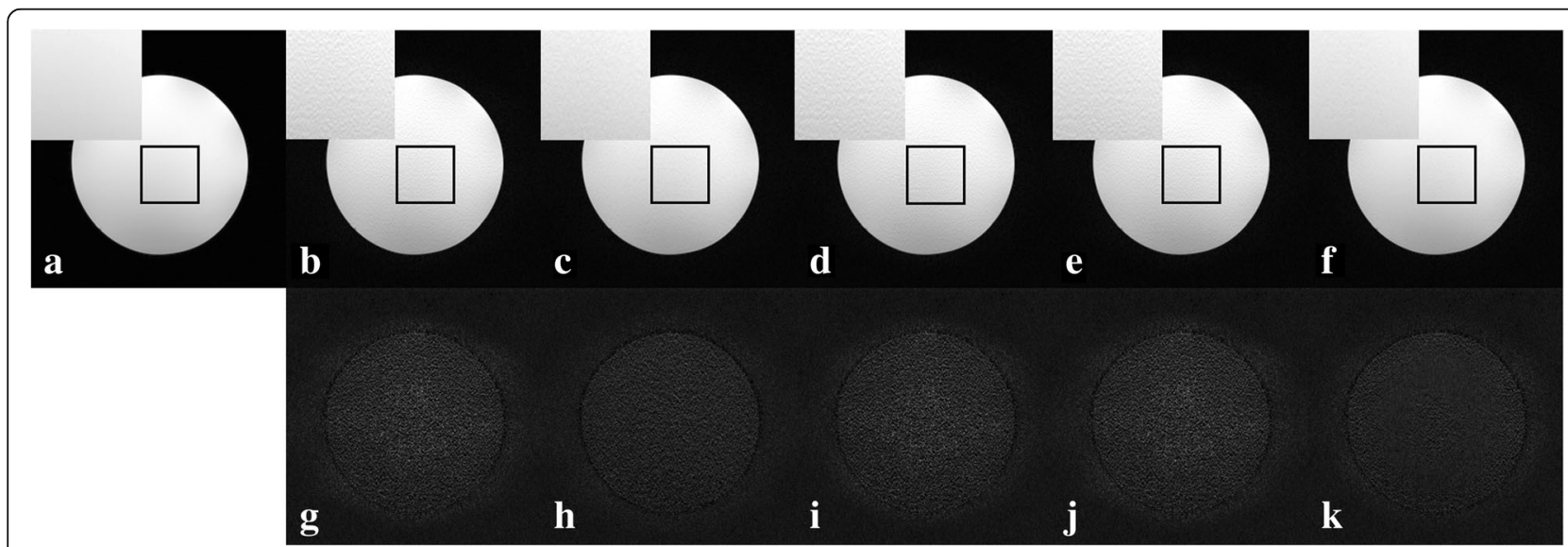

Fig. 2 Reconstructed images with ROI and difference maps of an eight-channel phantom data. The acquisition is accelerated with a net reduction factor of 2.29. a Reference image with SoS reconstruction. b GRAPPA reconstruction with 64 ACS lines and 4 outer reduction factor. $\mathbf{c}$ GRAPPA reconstruction with 40 ACS lines and 3 outer reduction factor. d Reconstruction using Tikhonov regularization. e Reconstruction using IRLS method. $\mathbf{f}$ Reconstruction using AV-GRAPPA. $\mathbf{g}-\mathbf{k}$ Difference maps of $\mathbf{b}-\mathbf{f}$, respectively. AV-GRAPPA is able to reconstruct the original reference image good. Other methods lose some fine structures and result in noise and aliasing artifacts 


\section{Results and discussion}

Figure 2 shows results of the reconstruction on phantom data set. The difference is noticeable among these methods. This experiment demonstrates that noised phantom can be recovered to high-quality image with less noise by the proposed method. Figures 3 and 4 show reconstruction results of both in vivo data sets. For fourchannel brain imaging, reconstructions by the proposed method achieve a good performance compared to other methods. For eight-channel brain imaging, the difference maps also show promising results as the previous data sets. The proposed method focuses on removing noise rather than suppressing aliasing artifacts, so the result of the proposed AV-GRAPPA still has tiny aliasing artifacts. It generally has the overall superior performance compared to other methods.
Table 1 shows the NMSEs for the reconstruction methods, in which columns represent different reduction factors, and rows denote different data sets. We fixed the kernel size as $4 \times 7$, and changed reduction factor for each data set. AV-GRAPPA generally generates less reconstruction error compared to the conventional GRAPPA and existing methods. We still set the number of the secondorder terms as 672, 784, and 896 for the phantom, fourchannel brain, and eight-channel brain data sets, respectively. The proposed AV-GRAPPA generally outperforms the other methods in terms of the NMSE measure. However, NMSE cannot describe the reconstructed image quality completely and accurately, because NMSE and other mean squared error measures do not correlate well with a subjective assessment of image quality. For eightchannel brain data reconstruction at reduction factor 6 ,

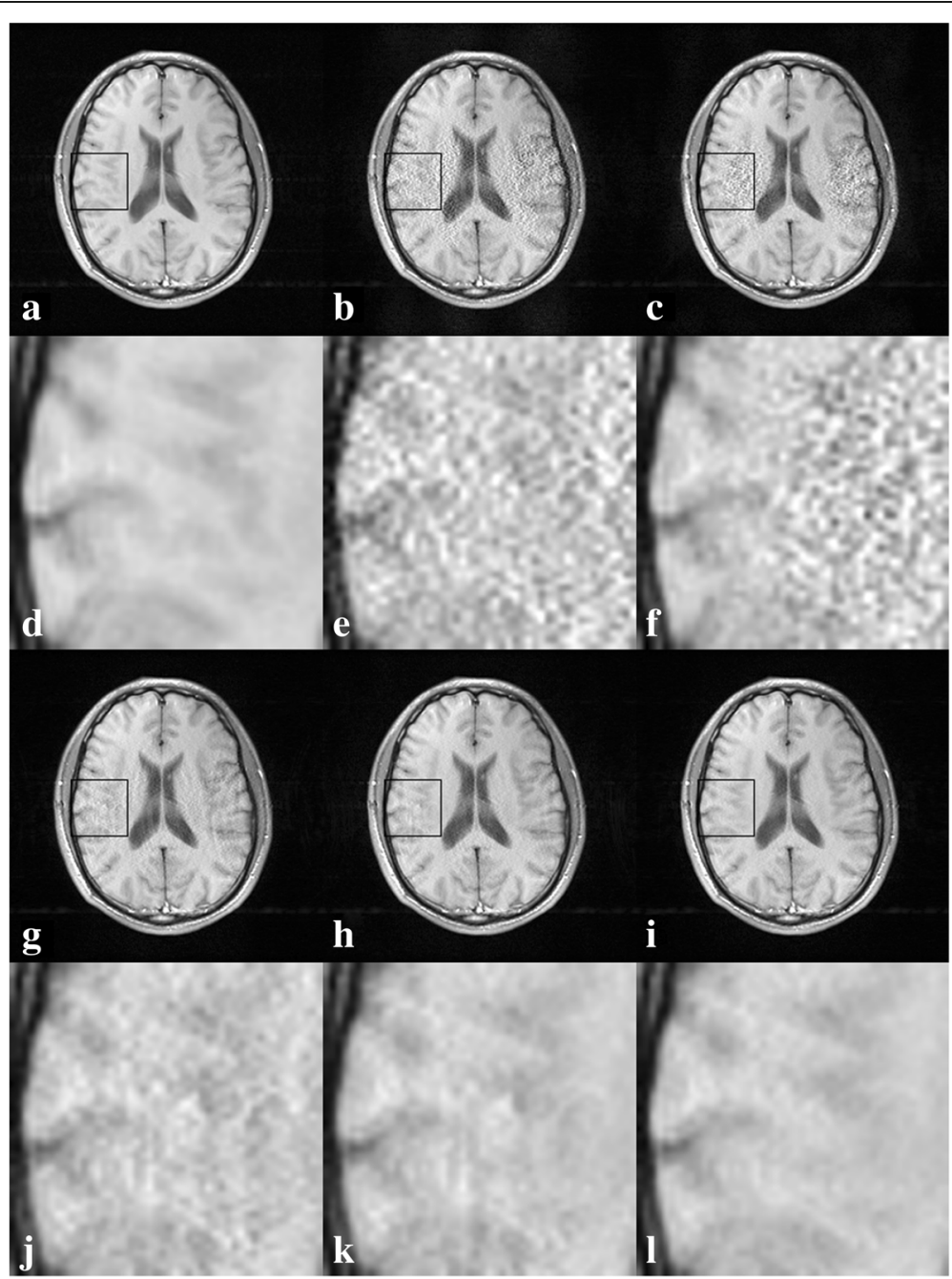

Fig. 3 Reconstructed images of a set of real four-channel axial brain data. The acquisition is accelerated with a net reduction factor of 2.56. a Reference image with SoS reconstruction. b GRAPPA reconstruction with 48 ACS lines and 4 outer reduction factor. c GRAPPA reconstruction with 22 ACS lines and 3 outer reduction factor. $\mathbf{d}$ Reconstruction using Tikhonov regularization. e Reconstruction using IRLS method. $\mathbf{f}$ Reconstruction using AV-GRAPPA. g-I Patches extracted from the same position in a-f, respectively. AV-GRAPPA is able to achieve high SNR while still keeping the image from blurring boundary. On the other hand, other methods reconstruct the image with noise more or less 


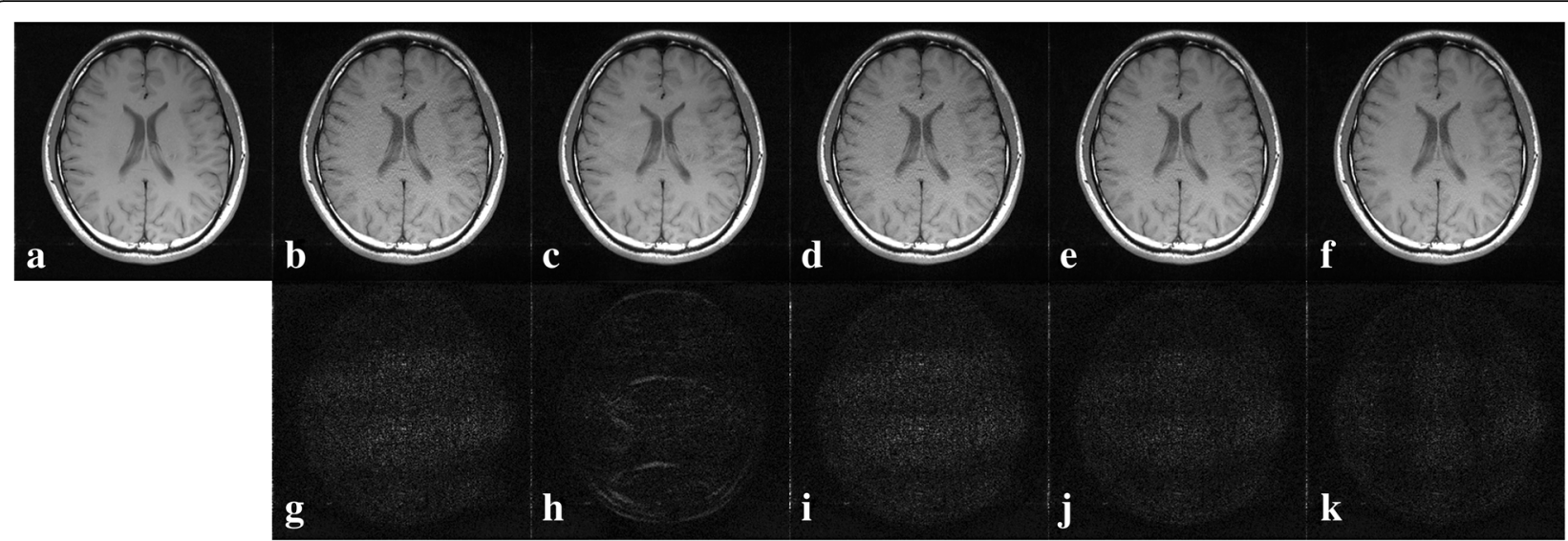

Fig. 4 Reconstructed images of a set of real eight-channel axial brain data. The acquisition is accelerated with a net reduction factor of 2.77. a Reference image with SoS reconstruction. b GRAPPA reconstruction with 38 ACS lines and 4 outer reduction factor. c GRAPPA reconstruction with 12 ACS lines and 3 outer reduction factor. $\mathbf{d}$ Reconstruction using Tikhonov regularization. e Reconstruction using IRLS method. $\mathbf{f}$ Reconstruction using AV-GRAPPA. g-k Difference maps of $\mathbf{b}-\mathbf{f}$, respectively. Similar to the results in Fig. 3, AV-GRAPPA reduces more noise than GRAPPA, Tikhonov regularization and IRLS method. In addition, since AV-GRAPPA has little ability to reduce aliasing artifacts, a few aliasing artifacts exist in the reconstructed image. But, aliasing artifacts also exist in other reconstructed images, compared to which, AV-GRAPPA keeps the aliasing artifacts at a relatively low level

reconstructed image by these methods has been seriously deteriorated by noise or artifacts, so NMSE measure cannot completely reflect the degree of deterioration. Although Tikhonov regularization and IRLS methods have lower NMSE value than that of the AV-GRAPPA, they reconstruct the image with high-level noise and aliasing artifacts. Another reason why the number of the second-order terms is so high for eight-channel brain data reconstruction at reduction factor 6 is that insufficient ACS lines result in deterioration in reconstruction, which is presented in the discussion section.

Since GRAPPA constitutes both fitting and interpolation processes, non-linear noise is generated in the final reconstructed $k$-space. Second-order Volterra filter is able to remove it to achieve high SNR, while the previously existing methods do not count the influence of non-linear noise. Tikhonov regularization presents good reconstruction results at low reduction factors. Although IRLS method can improve image quality to some extent, it still presents some noise. In the fitting process, small or zero weights are assigned to "outliers" in $k$-space, but these small or zero weights may be not accurate when they are used for estimating unacquired $k$-space signals in the interpolation.

This is one restriction of the AV-GRAPPA. The main reason is that second-order terms in adaptive Volterra filter will distort low-frequency estimation to some extent. As we know, magnitudes of signals at low-frequency are much larger than that of high-frequency. Outliers in lowfrequency regions will be enlarged by the second-order terms to distort the estimation of missing signals at lowfrequency estimation. On the other hand, it is capable of estimating weights more accurately for the high-frequency region in the $k$-space. For this reason, the proposed AVGRAPPA is good at suppressing noise and has little ability

Table 1 Quantitative evaluation by using NMSE measure is compared among reconstructed images of the conventional GRAPPA, Tikhonov regularization, IRLS, and the proposed AV-GRAPPA, which are denoted as " $G$," " $T$," "I," and " $V$ " in the table, respectively. " $R$ " represents the outer reduction factor, and the numbers of ACS lines are presented in the first column of the table. In addition, all reconstructions are implemented with the same sampling pattern. The proposed AV-GRAPPA generally has better performance than other methods

\begin{tabular}{|c|c|c|c|c|c|c|c|c|c|c|c|c|}
\hline \multirow[t]{2}{*}{ ACS } & \multicolumn{4}{|l|}{$R=4$} & \multicolumn{4}{|l|}{$R=5$} & \multicolumn{4}{|l|}{$R=6$} \\
\hline & $\overline{G(\%)}$ & $T(\%)$ & $1(\%)$ & $V(\%)$ & $G(\%)$ & $T(\%)$ & $/(\%)$ & $V(\%)$ & $G(\%)$ & $T(\%)$ & $1(\%)$ & $V(\%)$ \\
\hline & \multicolumn{12}{|c|}{ Phantom imaging } \\
\hline \multirow[t]{2}{*}{64} & 0.0637 & 0.0643 & 0.0637 & 0.0331 & 0.2204 & 0.1781 & 0.1756 & 0.0389 & 1.0312 & 0.2496 & 0.0755 & 0.0524 \\
\hline & \multicolumn{12}{|c|}{ Four-channel brain imaging } \\
\hline \multirow[t]{2}{*}{48} & 0.7283 & 0.1897 & 0.1195 & 0.0815 & 1.1265 & 0.3526 & 0.3878 & 0.2157 & 2.1861 & 0.5458 & 0.5207 & 0.4797 \\
\hline & \multicolumn{12}{|c|}{ Eight-channel brain imaging } \\
\hline 38 & 0.2063 & 0.2056 & 0.1379 & 0.1082 & 1.4566 & 0.7735 & 0.6847 & 0.2104 & 1.9476 & 0.7356 & 0.6448 & 2.2699 \\
\hline
\end{tabular}




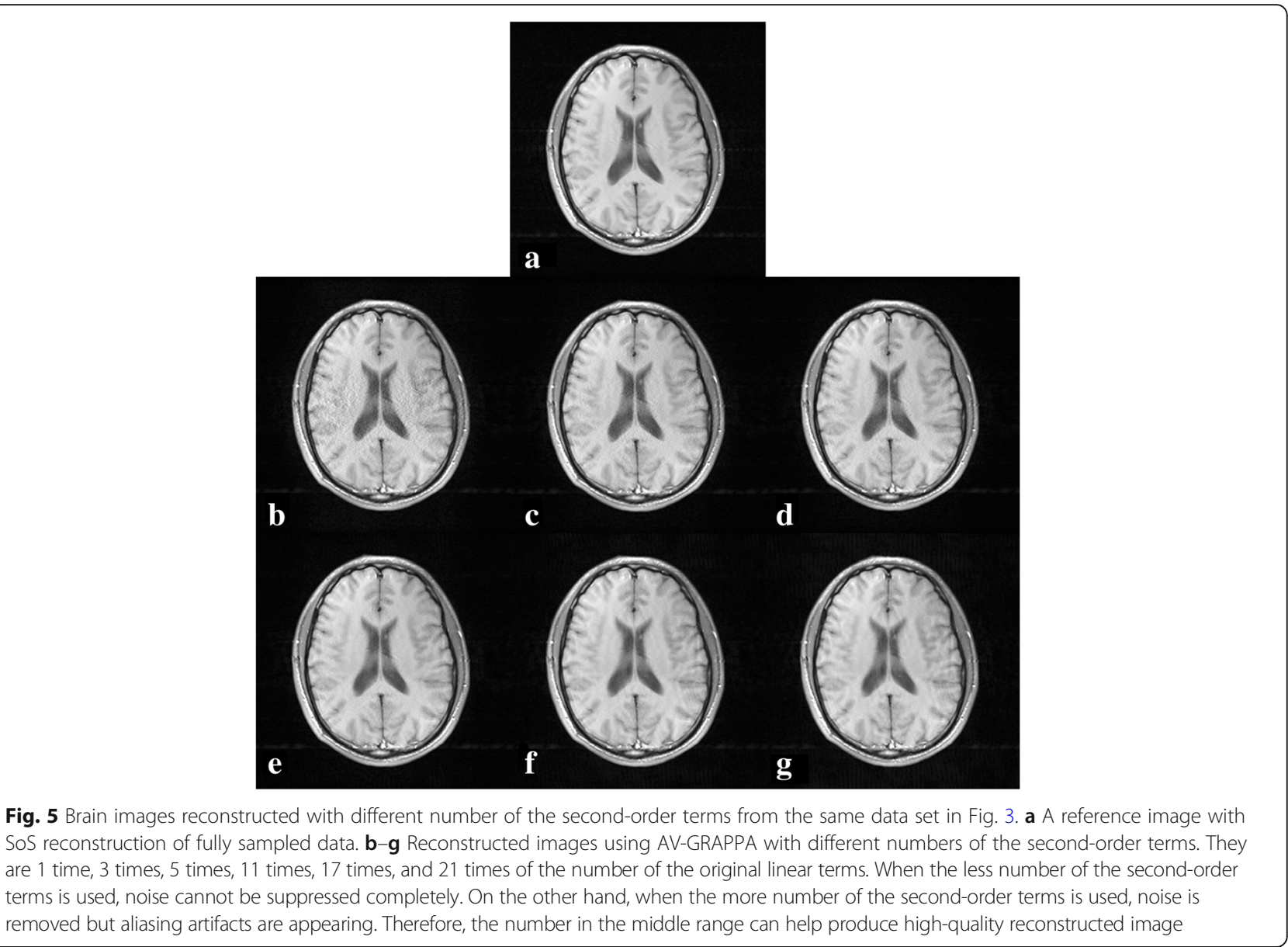

to reduce aliasing artifacts. Furthermore, higher-order Volterra filter (e.g., the third-order, the fourth-order Volterra filters) will deteriorate the reconstruction more seriously in our experiments. The reason contains two folds: only the second-order noise exists in the GRAPPA reconstruction so that the second-order Volterra filter should be used, and higher-order terms will distort the estimation of missing signals of low frequency aggravatingly.

Because the second-order part needs to be calculated by the adaptive Volterra filter compared to the conventional GRAPPA, in our experiments, reconstruction time is generally about 2-5 times of the conventional GRAPPA using the same convolution kernel size. Alternatively, the graph in Fig. 5 can help the user to tune the parameter $U$ to achieve the optimal reconstruction results if the computation time is not the crucial factor when using the AVGRAPPA.

\section{Conclusion}

A non-linear noise generation is analyzed in GRAPPA reconstruction. Based on the theory of Adaptive Volterra series, AV-GRAPPA is proposed in this paper. It contains second-order terms to suppress noise with the non-linear structure of the reconstruction system. The future research will focus on how to solve the problem of reconstruction quality through using a smaller number of ACS lines and attempt to combine the proposed method with the previous nonlinear gradient applications [23, 24]. Since the second-order terms can enlarge errors generated by outliers in the low-frequency region, how to find a way to avoid the estimation error to reduce aliasing artifacts is also a major goal.

\section{Abbreviations}

ACS: Auto-calibration signal; ANC: Active noise control; AV-GRAPPA: Adaptive Volterra generalized autocalibrating partial parallel acquisition; FIR: Finite impulse response; GRAPPA: Generalized autocalibrating partial parallel acquisition; IRLS: Iterative reweighted least-squares; MRI: Magnetic resonance imaging; NMSE: Normalized mean squared error; pMRI: Parallel magnetic resonance imaging; RF: Radio frequency; SAR: Specific absorption rate; SENSE: Sensitivity encoding; SMASH: Simultaneous acquisition of spatial harmonics; SNR: Signal-to-noise ratio; SoS: Sum of squares

\section{Acknowledgements}

The authors would like to acknowledge all the participants for their significant contributions to this research study.

Authors' contributions

$Y Z$, JL and $Y C$ conceived the idea of the study. $Y C$ designed the framework of the study. HW, YZ and SS collected the data. HW, YZ and YC carried out 
part of the algorithms. SS, ZH and JL analyzed the data and the results. HW and $Y C$ wrote the manuscript. YZ, SS, ZH and JL revised the manuscript. All authors read and approved the final manuscript.

\section{Funding}

This work was supported in part by the grant from the National Natural Science Foundation of China (nos. 61871373 and 81729003), Natural Science Foundation of Guangdong Province (no. 2018A0303130132), Sanming Project of Medicine in Shenzhen (no.SZSM201812005) and the Natural Science Foundation of Shenzhen (no. JCYJ20160331185933583).

\section{Availability of data and materials}

Data sharing is not applicable.

\section{Competing interests}

The authors declare that they have no competing interests.

\section{Author details}

'Paul C. Lauterbur Research Center for Biomedical Imaging, Shenzhen Institutes of Advanced Technology, Chinese Academy of Sciences, Shenzhen 518055, Guangdong, China. ${ }^{2}$ Medical Physics and Research Department, Hong Kong Sanatorium and Hospital, 2 Village Road, Happy Valley, Hong Kong SAR, China. ${ }^{3}$ Department of Neurology, Shenzhen Children's Hospital, Shenzhen 518038, Guangdong, China. ${ }^{4}$ Computer Science and Engineering Technology Department, University of Houston-Downtown, Houston, TX 77002, USA

Received: 28 February 2019 Accepted: 8 July 2019

Published online: 26 July 2019

\section{References}

1. D.W. McRobbie, E.A. Moore, M.J. Graves, M.R. Prince, MRI from Picture to Proton (Cambridge University Press, 2017)

2. E.M. Haacke, R.W. Brown, M.R. Thompson, R. Venkatesan, Magnetic resonance imaging: physical principles and sequence design, vol 82 (Wiley-Liss, New York, 1999)

3. G. Masselli, G. Gualdi, MR imaging of the small bowel. Radiology. 264(2), 333-348 (2012)

4. D.K. Sodickson, W.J. Manning, Simultaneous acquisition of spatial harmonics (SMASH): Fast imaging with radiofrequency coil arrays. Magn. Reson. Med. 38(4), 591-603 (1997)

5. K.P. Pruessmann, M. Weiger, M.B. Scheidegger, P. Boesiger, SENSE: Sensitivity encoding for fast MRI. Magn. Reson. Med. 42(5), 952-962 (1999)

6. M.A. Griswold, P.M. Jakob, R.M. Heidemann, M. Nittka, V. Jellus, J. Wang, B. Kiefer, A. Haase, Generalized autocalibrating partially parallel acquisitions (GRAPPA). Magn. Reson. Med. 47(6), 1202-1210 (2002)

7. J. Park, Q. Zhang, V. Jellus, O. Simonetti, D. Li, Artifact and noise suppression in GRAPPA imaging using improved k-space coil calibration and variable density sampling. Magn. Reson. Med. 53(1), 186-193 (2005)

8. R. Nana, T. Zhao, K. Heberlein, S.M. LaConte, X. Hu, Cross-validation-based kernel support selection for improved GRAPPA reconstruction. Magn. Reson. Med. 59(4), 819-825 (2008)

9. F. Huang, G. Duensing, in Proceedings of the Fourteenth Annual Meeting of the International Society of Magnetic Resonance in Medicine. A theoretical analysis of errors in GRAPPA (2006)

10. P.J. Shin, P.E. Larson, M.A. Ohliger, M. Elad, J.M. Pauly, D.B. Vigneron, M Lustig, Calibrationless parallel imaging reconstruction based on structured low-rank matrix completion. Magn. Reson. Med. 72(4), 959-970 (2014)

11. M. Uecker, P. Lai, M.J. Murphy, P. Virtue, M. Elad, J.M. Pauly, S.S. Vasanawala, M. Lustig, ESPIRiT_An eigenvalue approach to autocalibrating parallel MRI: Where SENSE meets GRAPPA. Magn. Reson. Med. 71(3), 990-1001 (2014)

12. C. Liao, Y. Chen, X. Cao, S. Chen, H. He, M. Mani, M. Jacob, V. Magnotta, J. Zhong, Efficient parallel reconstruction for high resolution multishot spiral diffusion data with low rank constraint. Magn. Reson. Med. 77(3), 1359-1366 (2017)

13. D. Liang, B. Liu, J. Wang, L. Ying, Accelerating SENSE using compressed sensing. Magn. Reson. Med. 62(6), 1574-1584 (2009)

14. P. Qu, C. Wang, G.X. Shen, Discrepancy-based adaptive regularization for GRAPPA reconstruction. J. Magn. Reson. Imaging 24(1), 248-255 (2006)

15. F. Lin, in Proc 14th Annual Meeting ISMRM, Seattle. Prior-regularized GRAPPA reconstruction (2006)
16. D. Huo, D.L. Wilson, Robust GRAPPA reconstruction and its evaluation with the perceptual difference model. J. Magn. Reson. Imaging 27(6), 1412-1420 (2008)

17. Z. Chen, J. Zhang, R. Yang, P. Kellman, L.A. Johnston, G.F. Egan, in 2009 IEEE International Conference on Control and Automation. 2D IIR filter for parallel magnetic resonance image reconstruction (IEEE, 2009), pp. 1792-1795

18. Z. Chen, J. Zhang, R. Yang, P. Kellman, L.A. Johnston, G.F. Egan, IIR GRAPPA for parallel MR image reconstruction. Magn. Reson. Med. 63(2), 502-509 (2010)

19. V. Volterra, Theory of functionals and of integral and integro-differential equations (Courier Corporation, 2005)

20. S.P. Boyd, Volterra series: Engineering fundamentals (1985)

21. R.D. Nowak, B.D. Van Veen, Random and pseudorandom inputs for Volterra filter identification. IEEE Trans. Signal Process. 42(8), 2124-2135 (1994)

22. J. Chani-Cahuana, C. Fager, T. Eriksson, Lower bound for the normalized mean square error in power amplifier linearization. IEEE Microwave Wireless Compon. Lett. 28(5), 425-427 (2018)

23. H. Wang, L. Tam, E. Kopanoglu, D.C. Peters, R.T. Constable, G. Galiana, Experimental O-space turbo spin echo imaging. Magn. Reson. Med. 75(4), 1654-1661 (2016)

24. H. Wang, L. Tam, E. Kopanoglu, D.C. Peters, R.T. Constable, G. Galiana, Ospace with high resolution readouts outperforms radial imaging. Magn. Reson. Imaging 37, 107-115 (2017)

\section{Publisher's Note}

Springer Nature remains neutral with regard to jurisdictional claims in published maps and institutional affiliations.

\section{Submit your manuscript to a SpringerOpen ${ }^{\circ}$ journal and benefit from:}

- Convenient online submission

- Rigorous peer review

- Open access: articles freely available online

High visibility within the field

- Retaining the copyright to your article

Submit your next manuscript at $\boldsymbol{\nabla}$ springeropen.com 